\title{
ANALGESIA TRANSOPERATÓRIA INDUZIDA PELA MORFINA OU MEPERIDINA EM GATOS SUBMETIDOS A OSTEOSSÍNTESE
}

\author{
TRANSOPERATIVE ANALGESIA INDUCED BY MORPHINE OR MEPERIDINE \\ IN CATS SUBMITTED TO OSTEOSYNTHESIS
}

\author{
João Marcelo Caldeira Cardoso Pinto da Cunha ${ }^{1}$ Silvia Renata Gaido Cortopassi ${ }^{2}$ \\ Aline Machado ${ }^{3}$
}

RESUMO

\begin{abstract}
$O$ objetivo do presente estudo foi avaliar a qualidade da analgesia proporcionada pela morfina e meperidina em gatos acometidos por processos traumáticos e submetidos a osteossíntese. Para tanto, foram utilizados 18 animais distribuídos em dois grupos: o grupo I recebeu acepromazina $(0,2 \mathrm{mg} / \mathrm{kg})$ e meperidina (4,0mg/kg), ambos pela via intramuscular; a indução anestésica foi realizada através da administração de quetamina $(3,0 \mathrm{mg} / \mathrm{kg})$ e midazolam $(0,3 \mathrm{mg} / \mathrm{kg})$ ambos pela via intravenosa, e a manutenção da anestesia foi efetuada com o auxílio de $100 \%$ de oxigênio e halotano. Os animais do grupo II foram pré-tratados com acepromazina $(0,2 \mathrm{mg} / \mathrm{kg})$ e morfina $(0,2 \mathrm{mg} / \mathrm{kg})$, ambos pela via intramuscular $e$ posteriormente foram submetidos ao mesmo tratamento proposto no grupo I. Foram avaliados: freqüência cardíaca e respiratória, pressão arterial sistólica, saturação da oxi-hemoglobina, concentração de dióxido de carbono no ar expirado, concentração inspirada e expirada do halotano, no período pré, trans, e pós-operatório. Concluiu-se que os fármacos opióides empregados apresentam propriedades adequadas para a utilização na medicação pré-anestésica de gatos que serão submetidos a procedimentos ortopédicos, uma vez que não foram observados sinais de depressão cardiovascular nem respiratória e a recuperação foi tranqüila e isenta de fenômenos excitatórios.
\end{abstract}

Palavras-chave: gatos, analgesia, opióides.

\section{SUMMARY}

The purpose of this study was to evaluate the quality of analgesia provided by morphine and meperidine in the cats presenting traumatic process and submitted to osteosynthesis, Eighteen animals were divided into two groups. Animals from group I were premedicated with acepromazine $(0.2 \mathrm{mg} / \mathrm{kg})$ and meperidine $(4.0 \mathrm{mg} / \mathrm{kg})$ and were induced with administration of ketamine $(3.0 \mathrm{mg} / \mathrm{kg})$ and midazolan $(0.3 \mathrm{mg} / \mathrm{kg})$. Anesthesia was mantained using halothane and $100 \%$ oxigen. Group II was

\begin{abstract}
premedicated with acepromazine $(0.2 \mathrm{mg} / \mathrm{kg})$ and morphine $(0.2 \mathrm{mg} / \mathrm{kg})$ and then submited to the same protocol proposed to group I. Heart and respiratory rate, systolic arterial blood pressure, oxihemoglobin satuation, carbon dioxide pressures, inspired and end-tidal concetrations of halothane were evaluated at pre, trans and pos surgery for statistical analysis, concluding that morfine and meperidine presented sastifactory analgesia.
\end{abstract}

Key words: cats, analgesia, opioids.

\section{INTRODUÇÃO}

A percepção da dor está associada a reações comportamentais e emocionais. A aplicação de estímulos nocivos no homem resulta no aumento da pressão arterial, da frequiência cardíaca, na dilatação pupilar e nas alterações nos parâmetros respiratórios. Quando essas mesmas respostas fisiológicas são vistas em animais, presume-se que há presença de dor (HEIDRICH \& KENT, 1985). Segundo LASCELLES e WATERMAN (1997), a dor causa sofrimento; reduz a ingestão de alimentos e do catabolismo, com ineficiente uso de energia; leva ao aumento do estresse, resultando atraso da cura do paciente; causa prejuízos à função respiratória com a ocorrência de hipóxia, hipercapnia e acidose; pode levar a automutilação e finalmente causa hipersensibilidade central, levando à dor crônica.

$\mathrm{O}$ gato geralmente permanece silencioso quando apresenta dor, mas pode rosnar quando abordado. Apresenta inapetência e tendência a se

\footnotetext{
${ }^{1}$ Acadêmico, Faculdade de Medicina Veterinária e Zootecnia (FMVZ), Universidade de São Paulo (USP).

${ }^{2}$ Médico Veterinário, Professor Doutor, FMVZ, USP, Departamento do Cirurgia, Av. Prof. Dr. Orlando Marques de Paiva, 87, Cidade Universitária, 05508-900, São Paulo, SP.

${ }^{3}$ Médico Veterinário, FMVZ, USP.
} 
esconder, sua postura é tensa, com apoio sobre o esterno e relutante a carícias. Um gato com dor intensa pode uivar e mostrar comportamento desesperado; lambidas freqüentes em um mesmo lugar também deve ser considerado um comportamento de dor (SANFORD et al., 1986).

Qualquer procedimento cirúrgico por mais superficial e pouco cruento que seja requer um adequado protocolo anestésico que promova analgesia durante o período peri-operatório. Dentre o grupo dos hipnoanalgésicos, a morfina é o fármaco padrão, embora inúmeros agentes analgésicos tenham sido sintetizados posteriormente, como é o caso da meperidina. Seu emprego é indicado em qualquer situação na qual se deseja obter alívio da dor: seja no período pré-anestésico ou no transoperatório. Este agente distribui-se pelos diferentes tecidos, em particular, atinge o sistema nervoso central, fígado, rins, pulmões e músculos (GORNIAK, 1996). No sistema respiratório, causa depressão em virtude de sua ação direta sobre o centro respiratório, podendo ocorrer também a produção de respiração regular e periódica (SHORT, 1987). A depressão respiratória máxima ocorre com aproximadamente sete minutos após a administração intravenosa de morfina, e cerca de pelo menos 30 minutos após administração intramuscular ou 90 minutos após a administração subcutânea. A sensibilidade do centro respiratório começa a retornar ao normal com duas a três horas, entretanto o volume respiratório ainda se mantém diminuído por quatro a cinco horas depois da administração da dose terapêutica (SHORT, 1987). A constipação intestinal é exacerbada pela redução das secreções biliares, gástrica e pancreática. A náusea e o vômito ocasionados pela morfina estão relacionados à estimulação da zona deflagradora dos quimiorreceptores localizadas nas paredes laterais do terceiro ventrículo (área postrema). No gato, há necessidade de uma dose elevada para produzir o vômito (GÓRNIAK, 1996). Segundo WATTS et al. (1973), gatos tratados com $0,1 \mathrm{mg} / \mathrm{kg}$ pela via subcutânea, apresentaram boa analgesia e tranqüilização aparente. Quando utilizadas doses de $1,0 \mathrm{mg} / \mathrm{kg}$ não ocorre aparente aumento do efeito analgésico e narcótico. Já em doses de $10 \mathrm{mg} / \mathrm{kg}$, há o maior grau de analgesia, mas há o aparecimento de efeitos tóxicos com hipermotilidade, ataxia, midríase, salivação e intolerância a barulhos.

$\mathrm{O}$ efeito analgésico da morfina é de 1 a 4 horas, quando administrada por via intravenosa ou de 2 a 6 horas, quando administrada por via intramuscular (HELLYER \& GAYNOR, 1998).

Em relação a meperidina, inicialmente foi introduzida na clínica como agente espasmolítico do tipo atropina. Mais tarde, constatou-se que este fár- maco tinha efeito hipnoanalgésico semelhante ao da morfina, porém com potência analgésica dez vezes menor. Promove também menor atividade hipnótica, efeito constipante e ação sobre o centro da tosse, quando comparada à morfina (GÓRNIAK, 1996). Possui larga margem de segurança e é um opióide muito utilizado em gatos (TRIM, 1987). O início do efeito analgésico após a administração subcutânea ou intramuscular ocorre ao redor de 10 minutos, alcançando o pico após uma hora (SHORT, 1987). Depois da administração da meperidina por via intravenosa, há aumento no fluxo sangüíneo periférico e diminuição da circulação arteriolar e da resistência periférica (SHORT, 1987). Segundo DYSON (1990), o uso de meperidina pela via intravenosa promove a liberação de histamina, fato não observado com o butorfanol. O uso clínico, a duração e as ações são menores, se comparadas com a morfina. A depressão respiratória é semelhante a da morfina, ocorrendo o máximo de depressão uma hora após a administração do fármaco (SHORT, 1987). Em doses terapêuticas, a meperidina não possui significantes efeitos inconvenientes no sistema cardiovascular (TRIM, 1987).

É importante salientar que o reconhecimento da dor nos animais requer uma interpretação complexa dos comportamentos apresentados pelo animal. Parte desta dificuldade na caracterização dos processos dolorosos está na dependência da avaliação de parâmetros subjetivos.

As reações crônicas de dor podem ser sutis e incluem anorexia, mudança de comportamento, claudicação e agitação (SMITH et al., 1996). As respostas fisiológicas da dor são taquipnéia, taquicardia com ou sem arritmia, midríase, atelectasia e salivação (WERNER \& TABOADA, 1994). Pouco se conhece sobre os comportamentos apresentados pelos gatos quando apresentam dor ou estresse cirúrgico. Sabe-se que os gatos têm tendência a se esconderem e tornarem-se mais quietos do que o normal, ou apresentar posturas rígidas ou encurvadas (WERNER \& TABOADA, 1994).

Desta forma, o objetivo do presente estudo foi avaliar a qualidade da analgesia proporcionada pela morfina e meperidina no período transoperatório de processos ortopédicos, observada através de parâmetros vitais e pela concentração expirada do anestésico inalatório, e no período pósoperatório imediato, através de alguns escores propostos, efetuando-se assim, comparação entre estes dois fármacos.

\section{MATERIAL E MÉTODO}

Foram utilizados 18 gatos, provenientes do Serviço de Cirurgia do Departamento de Cirurgia 
da Faculdade de Medicina Veterinária e Zootecnia da Universidade de São Paulo, acometidos de processo traumático e que foram submetidos à osteossíntese. Foram 12 machos e seis fêmeas, 14 animais sem raça definida e quatro com raça definida, variando entre quatro a 24 meses de idade e entre 1,95 a 4,30 quilos de peso vivo. Os animais foram distribuídos aleatoriamente e de modo cego simples em dois grupos: o grupo I recebeu $0,2 \mathrm{mg} / \mathrm{kg}$ de acepromazina $^{\mathrm{d}}$ e $4,0 \mathrm{mg} / \mathrm{kg}$, de meperidina ${ }^{\mathrm{f}}$ através da via intramuscular; o grupo II recebeu $0,2 \mathrm{mg} / \mathrm{kg}$ de acepromazina ${ }^{\mathrm{d}}$ e $0,2 \mathrm{mg} / \mathrm{kg}$ de morfina ${ }^{\mathrm{e}}$ através da via intramuscular. Decorridos 20 minutos, nos gatos de ambos os grupos, a indução da anestesia foi realizada através da administração de quetamina $^{\mathrm{g}}$ e midazolam ${ }^{\mathrm{h}}$, respectivamente nas doses de $3,0 \mathrm{mg} / \mathrm{kg}$ e $0,3 \mathrm{mg} / \mathrm{kg}$, ambos através da via intravenosa. Uma vez que os animais apresentaram relaxamento da região mandibular e ausência do reflexo laringotraqueal, foi realizada a intubação orotraqueal com sonda de diâmetro adequando, e a mesma foi conectada ao circuito de anestesia Baraka. Através deste, os animais receberam halotano ${ }^{i}$ em oxigênio a $100 \%$ na concentração que permitisse a manutenção do $3^{\circ}$ plano de anestesia. Durante o procedimento cirúrgico, os animais receberam solução de Ringer com Lactato na taxa de $5,0 \mathrm{~m} \ell / \mathrm{kg} / \mathrm{hora}$.

Foram avaliados os seguintes parâmetros: freqüência cardíaca (através do monitor cardíaco ${ }^{a}$ ); pressão arterial sistólica (com auxílio de estetoscópio ultrassônico Doppler ${ }^{\mathrm{b}}$ ); frequiência respiratória (através da verificação dos movimentos da caixa torácica); saturação periférica da oxihemoglobina (através do posicionamento do sensor do oxímetro de pulso ${ }^{\mathrm{b}}$ na língua, membrana interdigital ou orelha desprovida de pêlo e de pigmentação); concentração de dióxido de carbono no ar expirado (mensurado continuamente com capnográfo $^{c}$ sendo o sensor posicionado na porção expiratória da peça $\mathrm{T}$ de Ayres); concentração inspirada e expirada do halotano (de forma contínua através do analisador de gases ${ }^{c}$ ).

Os parâmetros fisiológicos foram mensurados a cada cinco minutos. Para fins de análise estatística, os parâmetros analisados foram aqueles obtidos nos seguintes momentos: imediatamente antes da medicação pré-anestésica; 15 minutos após a medicação pré-anestésica; aos cinco, 15, 30 e 60 minutos após a indução da anestesia; no momento da extubação e aos 30 e 60 minutos após a extubação. A saturação da oxi-hemoglobina, a concentração de dióxido de carbono no ar expirado e a concentração inspirada e expirada do anestésico inalatório só fo- ram mensurados após a indução da anestesia (cinco, 15,30 e 60 minutos).

Através da avaliação pós-operatória, foi possível avaliar a qualidade da recuperação do animal e mensurar o grau de analgesia promovido. Esta mensuração foi realizada através de atributos propostos por HELLYER \& GAYNOR (1998). A avaliação pós-operatória foi realizada nos seguintes momentos: logo após o término do procedimento cirúrgico (animal extubado) e decorridos 30 e 60 minutos do primeiro exame pós-operatório executado.

Os valores obtidos foram avaliados pela ANOVA (análise de variância), seguida do teste de Tukey para a comparação dos diferentes tempos de observação de um mesmo grupo. Para a comparação entre os dois grupos experimentais foi utilizado o teste $\mathrm{T}$ de Student. O grau de significância estabelecido para os dois testes estatísticos foi de 5\% $(\mathrm{p}<0,05)$. Através da prova de Mann-Whitney "U", foi realizada a comparação entre os dois grupos experimentais na avaliação pós-operatória imediata da análise. Os três testes estatísticos foram realizados em programa de computador. ${ }^{\mathrm{j}}$

\section{RESULTADOS}

Os resultados da avaliação dos parâmetros mensurados nos períodos pré, trans e pós-operatório estão apresentados na tabela 1 . Não foi observada diferença estatisticamente significativa entre os pesos e as idades dos animais dos grupos I e II. No estudo comparativo entre os dois grupos apenas os valores de pressão arterial sistólica (PAS) apresentaram diferença significativa, sendo que nos momentos da extubação, 30 e 60 minutos após a extubação, os animais do grupo II apresentaram valores superiores aos do grupo I.

O período de cirurgia foi de $101,1 \pm 23,5 \mathrm{~min}$ no grupo I e $104,4 \pm 33,4 \mathrm{~min}$ no grupo II, sem diferença estatística entre os mesmos.

$\mathrm{Na}$ avaliação individual do grupo I, observou-se que a frequiência respiratória obtida aos 5 minutos após a indução foi inferior à avaliada aos 30 e 60 minutos após a extubação. Em relação à concentração de halotano no ar inspirado expirado, ambos os valores observados no momento da extubação foram inferiores aos obtidos aos cinco, 15 e 30 minutos de anestesia.

$\mathrm{Na}$ avaliação individual do grupo II, observou-se que a freqüência cardíaca, avaliada aos 60 minutos após a indução anestésica, foi inferior a avaliadas após 15 minutos da medicação préanestésica $(\mathrm{p}<0,01)$ e após 5 minutos da indução anestésica $(\mathrm{p}<0,01)$. A freqüência respiratória obser- 
Tabela 1 - Valores médios e desvios-padrões da frequiência cardíaca (FC) (batimentos por minuto), frequiência respiratória (FR) (movimentos respiratórios por minuto), pressão arterial sistólica (PAS) $(\mathrm{mmHg})$, concentração de dióxido de carbono no ar expirado (ETCO $\left.{ }_{2}\right)$ $(\mathrm{mmHg})$, saturação periférica da oxi-hemoglobina $\left(\mathrm{SpO}_{2}\right)(\%)$ e concentração de halotano (\%) inspirado e expirado de gatos tratados com acepromazina/meperidina (GI) e acepromazina/morfina (GII) nos diversos momentos de avaliação.

\begin{tabular}{|c|c|c|c|c|c|c|c|c|c|c|}
\hline \multirow[t]{2}{*}{ Parâmetros } & \multirow[t]{2}{*}{ Grupos } & \multirow[t]{2}{*}{ Pré-MPA } & \multirow{2}{*}{$\begin{array}{c}\text { 15min pós- } \\
\text { MPA }\end{array}$} & \multicolumn{4}{|c|}{ Min após indução da anestesia } & \multirow[t]{2}{*}{ Extubação } & \multicolumn{2}{|c|}{ Min pós-extubação } \\
\hline & & & & 5 & 15 & 30 & 60 & & 30 & 60 \\
\hline $\mathrm{FC}(\mathrm{bpm})$ & $\begin{array}{l}\text { I } \\
\text { II }\end{array}$ & $\begin{array}{l}151,3 \pm 28,8 \\
170,8 \pm 16,2\end{array}$ & $\begin{array}{l}163,6 \pm 30,4 \\
179,6 \pm 20,9\end{array}$ & $\begin{array}{l}161,0 \pm 29,8 \\
173,9 \pm 26,8\end{array}$ & $\begin{array}{l}142.1 \pm 28.3 \\
151.3 \pm 28.9\end{array}$ & $\begin{array}{l}135.7 \pm 22.1 \\
148.7 \pm 21.7\end{array}$ & $\begin{array}{l}132.1 \pm 26.7 \\
135.2 \pm 23\end{array}$ & $\begin{array}{l}151.3 \pm 26.7 \\
153.3 \pm 25\end{array}$ & $\begin{array}{r}162.7 \pm 33.4 \\
171 \pm 26.3\end{array}$ & $\begin{array}{l}160.7 \pm 28.7 \\
169.3 \pm 24.5\end{array}$ \\
\hline FR (mpm) & $\begin{array}{l}\text { I } \\
\text { II }\end{array}$ & $\begin{array}{l}40,9 \pm 5,3 \\
46,8 \pm 15,7\end{array}$ & $\begin{array}{l}33,8 \pm 9,0 \\
37,0 \pm 10,0\end{array}$ & $\begin{array}{l}14,4 \pm 5,5^{@} \\
23,6 \pm 14,2^{@}\end{array}$ & $\begin{array}{l}18,7 \pm 12,1 \\
27,4 \pm 13,1\end{array}$ & $\begin{array}{l}26,9 \pm 17,0 \\
33,4 \pm 18,5\end{array}$ & $\begin{array}{l}23,3 \pm 13,3 \\
29,7 \pm 13,3\end{array}$ & $\begin{array}{l}35,1 \pm 27,2 \\
30,4 \pm 14,9\end{array}$ & $\begin{array}{l}39,8 \pm 15,4 \\
42,2 \pm 10,6\end{array}$ & $\begin{array}{c}38,0 \pm 15,5 \\
39,9 \pm 9,5\end{array}$ \\
\hline PAS (mmHg) & $\begin{array}{l}\text { I } \\
\text { II }\end{array}$ & $\begin{array}{l}133,9 \pm 31,3 \\
134,0 \pm 25,8\end{array}$ & $\begin{array}{l}115,3 \pm 17,3 \\
118,4 \pm 20,0\end{array}$ & $\begin{array}{r}102,1 \pm 28,8 \\
89,4 \pm 23,6\end{array}$ & $\begin{array}{l}99,1 \pm 21,3 \\
85,3 \pm 23,5^{@}\end{array}$ & $\begin{array}{l}106,1 \pm 25,4 \\
104,2 \pm 24,8\end{array}$ & $\begin{array}{c}94,3 \pm 21,1 \\
98,4 \pm 41,7\end{array}$ & $\begin{array}{l}122,0 \pm 40,3^{*} \\
166,5 \pm 27,8\end{array}$ & $\begin{array}{c}130,3 \pm 25,4^{*} \\
167,7 \pm 29,0\end{array}$ & $\begin{array}{l}136,9 \pm 17,5^{*} \\
160,6 \pm 16,7\end{array}$ \\
\hline $\begin{array}{l}\mathrm{ETCO} 2 \\
(\mathrm{mmHg})\end{array}$ & $\begin{array}{l}\text { I } \\
\text { II }\end{array}$ & - & - & $\begin{array}{l}32,3 \pm 9,8 \\
28,4 \pm 7,6\end{array}$ & $\begin{array}{l}28,6 \pm 6,5 \\
28,4 \pm 8,9\end{array}$ & $\begin{array}{l}27,7 \pm 8,6 \\
23,3 \pm 7,8\end{array}$ & $\begin{array}{l}28,3 \pm 7,9 \\
27,4 \pm 6,2\end{array}$ & $\begin{array}{l}27,4 \pm 5,3 \\
23,3 \pm 7,1\end{array}$ & - & $\begin{array}{l}- \\
-\end{array}$ \\
\hline $\mathrm{SpO} 2 \%$ & $\begin{array}{l}\text { I } \\
\text { II }\end{array}$ & $\begin{array}{l}- \\
-\end{array}$ & - & $\begin{array}{l}94,8 \pm 4,3 \\
94,6 \pm 3,1\end{array}$ & $\begin{array}{r}97 \pm 1,5 \\
96,1 \pm 2,3\end{array}$ & $\begin{array}{l}96,7 \pm 1,4 \\
94,3 \pm 4,0\end{array}$ & $\begin{array}{l}97,3 \pm 0,8 \\
95,3 \pm 4,9\end{array}$ & $\begin{array}{l}95,9 \pm 1,5 \\
93,6 \pm 4,2\end{array}$ & $\begin{array}{l}- \\
-\end{array}$ & $\begin{array}{l}- \\
-\end{array}$ \\
\hline $\begin{array}{l}\text { Halotano exp } \\
(\%)\end{array}$ & $\begin{array}{l}\text { I } \\
\text { II }\end{array}$ & $\begin{array}{l}- \\
-\end{array}$ & - & $\begin{array}{l}1,2 \pm 0,5 \\
1,5 \pm 0,9\end{array}$ & $\begin{array}{l}1,3 \pm 0,6 \\
1,5 \pm 1,2\end{array}$ & $\begin{array}{l}1,0 \pm 0,2 \\
1,3 \pm 0,6\end{array}$ & $\begin{array}{l}0,8 \pm 0,3 \\
1,0 \pm 0,4\end{array}$ & $\begin{array}{l}0,3 \pm 0,2 \\
0,1 \pm 0,1\end{array}$ & $\begin{array}{l}- \\
-\end{array}$ & $\begin{array}{l}- \\
-\end{array}$ \\
\hline $\begin{array}{l}\text { Halotano insp } \\
(\%)\end{array}$ & $\begin{array}{l}\text { I } \\
\text { II }\end{array}$ & $\begin{array}{l}- \\
-\end{array}$ & - & $\begin{array}{l}1,9 \pm 1,6 \\
2,1 \pm 1,2\end{array}$ & $\begin{array}{l}1,7 \pm 0,8 \\
1,7 \pm 1,5\end{array}$ & $\begin{array}{l}1,2 \pm 0,4 \\
1,4 \pm 0,6\end{array}$ & $\begin{array}{l}1,1 \pm 0,3 \\
1,0 \pm 0,4\end{array}$ & $\begin{array}{l}0,2 \pm 0,3 \\
0,0 \pm 0,1\end{array}$ & $\begin{array}{l}- \\
-\end{array}$ & $\begin{array}{l}- \\
-\end{array}$ \\
\hline
\end{tabular}

MPA - medicação pré-anestésica; exp - expirado; insp - inspirado.

@ $\mathrm{p}<0,05$ em relação aos valores basais; $\mathrm{p}<0,05^{*}$ entre os grupos I e II.

vada aos 5 minutos após indução foi inferior aos valores basais, sendo a diferença estatisticamente significativa $(\mathrm{p}<0,05)$.

Em relação à pressão arterial sistólica, houve uma redução da PAS no decorrer da anestesia, sendo que os valores obtidos aos 15 minutos da anestesia inalatória foram inferiores em relação aos valores basais $(\mathrm{p}<0,05)$. Os observados no momento da extubação e aos 30 e 60 minutos após a extubação foram superiores aos avaliados aos cinco, 15, 30 e 60 minutos após a indução anestésica (sendo $\mathrm{p}<0,001 \mathrm{em}$ todos os momentos).

As concentrações de halotano no ar inspirado e expirado observadas no momento da extubação foram inferiores aos valores observados aos cinco, 15 e 30 minutos após a indução anestésica (respectivamente $\mathrm{p}<0,01, \mathrm{p}<0,01 \mathrm{e} \mathrm{p}<0,05)$.

Os escores observados durante a avaliação pós-operatória, nos diferentes momentos não apresentaram diferença significativa tanto na análise comparativa quanto na análise individual entre os dois grupos.

\section{DISCUSSÃO}

Em consideração a dor pós-operatória, BONICA (1992) menciona que, no homem, o controle da dor tem sido e continua a ser pouco eficiente e, conseqüentemente, de 40 a $75 \%$ dos pacientes continuam a ter dores de moderada a intensa. Em adição ao sofrimento, a dor tem contribuído diretamente e indiretamente nas complicações no pós-operatório e conseqüente aumento da mortalidade, prolongando a convalescença e aumentando o tempo de hospitalização. Com base em revisões bibliográficas de literaturas veterinárias, verificou-se que a situação é idêntica, não sendo melhor e realmente é provável que seja pior nos animais submetidos a procedimentos cirúrgicos com propósito experimental ou curativo. $\mathrm{O}$ conceito de analgesia profilática implica a administração do analgésico previamente ao insulto doloroso, ou antes, que o processo inflamatório causado pelo trauma cirúrgico já esteja instalado. Diversos autores têm sugerido que o pré-tratamento com o uso de fármacos analgésicos diminui a dor pós-operatória (McQUAY, 1992; BRIDENBAUGH, 1994). O grupo de fármacos que podem induzir analgesia profilática compreende os opióides, alfa 2 agonistas, antiinflamatórios não esteroidais e anestésicos locais. O emprego isolado ou a associação de fármacos depende do tipo de dor, duração do efeito, disponibilidade do fármaco e eventuais efeitos colaterais que possam ser induzidos.

A escolha da meperidina e da morfina no pré-operatório de felinos submetidos a osteossíntese ocorreu basicamente devido à potência analgésica de ambos os fármacos assim como a adequada duração de efeito desencadeada pelos dois agentes.

Segundo BONAGURA (1994), os valores fisiológicos de freqüência cardíaca nos felinos domésticos estão situados entre 90 e 120 batimentos por minuto (bpm) nos animais tranqüilos e entre 140 
e 220bpm no animal sob exame clínico. Sendo assim, os valores obtidos no exame pré-anestésico nos dois grupos podem ser considerados normais (médias de 151,3 e 170,8 respectivamente nos grupos I e II), uma vez que estes animais apresentavam ansiedade e, algumas vezes, desconforto. Com emprego do halotano, há diminuição da FC dose-dependente (PADDLEFORD, 1999), o que explica a redução verificada nos dois grupos estudados.

A redução da pressão arterial sistólica apresentada pelos animais do grupo II durante a manutenção da anestesia, deve-se provavelmente aos efeitos depressores promovidos pelo halotano. Este agente anestésico reduz o débito cardíaco em cerca de 20 a $40 \%$, além de induzir importante diminuição da resistência vascular periférica (FANTONI $\boldsymbol{e t}$ al., 1999). A hipotensão arterial também poderia ser causada pela administração intravenosa da meperidina ou da morfina (THURMON et al., 1996), porém, no experimento em tela, os dois fármacos foram empregados através da via intramuscular.

Aos 30 e 60 minutos pós extubação, os animais do grupo II apresentaram valores de PAS ligeiramente elevados em relação aos do grupo I, porém ambos dentro do padrão de normalidade. Isso poderia estar relacionado à recuperação mais rápida destes animais (já na extubação apresentavam valores mais elevados) uma vez que a concentração expirada de halotano no momento da extubação era inferior.

Os valores da frequiência respiratória, no momento que sucedeu a indução, podem estar relacionados com a depressão respiratória, causada pelos opióides (SHORT, 1987; THURMON et al., 1996) assim como ação depressora temporária do midazolam (MAMA, 1998). Além disto, nos planos iniciais do III Estágio de Guedel, é esperada a diminuição da frequiência respiratória quando anestésicos halogenados são utilizados (FANTONI et al., 1999).

Vale lembrar que, no início da manutenção da anestesia inalatória, fez-se necessária a ventilação assistida, uma vez que os felinos apresentavam-se em apnéia, apesar do odor agradável (FANTONI et al., 1999). Embora tenha ocorrido redução da freqüência respiratória, em alguns momentos de avaliação, os gatos de ambos os grupos não apresentaram incremento nos valores de dióxido de carbono expirado, demonstrando que a bradipnéia não induziu hipoventilação.

Apesar de a indução da analgesia e a recuperação anestésica terem sido satisfatórias, o emprego prévio dos dois analgésicos não acarretou qualquer diminuição significativa na concentração do anestési- co inalatório empregado, quando se levou em consideração o valor da concentração alveolar mínima do halotano no gato (1,2) (FANTONI et al., 1999).

A avaliação de dor em animais é bastante subjetiva, porém alguns escores e escalas têm sido desenvolvidos com intuito de aprimorar este parâmetro. LASCELLES e WATERMAN (1997) desenvolveram pontuação baseada nos sinais de desconforto e presença ou não de reação frente à pressão sobre a área lesada. Uma outra forma de avaliar a analgesia pós-operatória é através da escala analógica visual onde zero representa ausência de dor e dez, a pior dor imaginável (GOMES do AMARAL et al., 1997).

O método utilizado baseou-se no proposto por HELLYER e GAYNOR (1998), em que se avaliou o conforto, movimento, aparência, comportamento interativo, vocalização e frequiências cardíaca e respiratória. Apenas um gato do grupo I (animal $\mathrm{n}^{\mathrm{o}} 5$ ) apresentou escore acima de 15 no momento da extubação, porém se acredita que este valor esteja relacionado com a agitação decorrente da superficialização da anestesia no momento da extubação, uma vez que posteriormente, os escores foram inferiores (13 e 11). Os resultados obtidos demonstraram que os dois grupos apresentaram analgesia similar nos três momentos de avaliação.

A partir dos resultados pode-se concluir que os fármacos empregados apresentam propriedades adequadas para a utilização na medicação préanestésica de gatos que serão submetidos a procedimentos ortopédicos, uma vez que não foram observados sinais de depressão cardiovascular nem respiratória, apesar de não reduzirem a concentração expirada do anestésico inalatório. Além disso, o período hábil dos agentes foi apropriado para procedimentos de média duração visto que a recuperação foi tranqüila e isenta de fenômenos excitatórios.

\section{FONTES DE AQUISIÇÃO}

${ }^{\text {a }}$ Monitor de eletrocardiograma DX-920 Dixtal

${ }^{\mathrm{b} O x i ́ m e t r o ~ d e ~ P u l s o ~-~ B i o c h e n ~}$

${ }^{\mathrm{c}}$ Multinex - Analisador de gases anestésicos e capnógrafo -

Datascope

${ }^{\mathrm{d}}$ Acepran a $0,2 \%$ - Univet S.A.

${ }^{\text {e}}$ Dimorf - Lab. Cristália

${ }^{\mathrm{f}}$ Dolantina - Hoescht do Brasil Química e Farmacêutica

${ }^{\mathrm{g}}$ Ketalar - Parke davis - Achè Laboratórios Farmacêuticos S.A.

${ }^{\text {h}}$ Dormonid - Produtos Roche Químicos e Farmacêuticos S.A.

${ }^{\mathrm{i}}$ Halotano - Hoescht do Brasil Química e Farmacêutica

${ }^{\mathrm{j}}$ Instat - Graphpad Software

${ }^{\mathrm{I}}$ Doppler - Imbracrios -São Paulo

\section{REFERÊNCIAS BIBLIOGRÁFICAS}

BONAGURA, J.D. Cardiovascular diseases. In: SHERDING, R.G. The cat disease and clinical management. Philadelphia : Saunders, 1994. Cap.31, p.819-946. 
BONICA, J.J. Pain research and therapy: History, current status and future goals. In: SHORT, E.C.; POZNAK, A.V. Animal pain. Nova York : Churchill Livingstone, 1992. Cap.1, p.2021.

BRIDENBAUGH, P.O. Preemptive analgesia - is it clinically relevant? Anesthesia and Analgesia, v.78, n.1, p.203-204, 1994.

FANTONI, D.T.; CORTOPASSI, S.R.G.; BERNARDI, M.M. Anestésicos inalatórios. In: SPINOSA, H.S.; GÓRNIAK, S.L.; BERNARDI, M.M. Farmacologia aplicada à medicina veterinária. 2. ed. Rio de Janeiro : Guanabara Koogan, 1999. Cap.10. p.103-113.

GOMES do AMARAL, J.L.; JOAQUIM, M.R.G.; RODRIGUES Jr., G.R., et al. Analgesia. In: GOMES do AMARAL, J.L. Sedação, analgesia e bloqueio neuromuscular em UTI São Paulo : Atheneu, 1997. Cap.3, p.47-75.

GORNIAK, S.L. Hipnoanalgésicos e neuroleptoanalgesia. In: SPINOSA, V.; GÓRNIAK, S.L.; BERNARDI, M.M. Farmacologia aplicada à medicina veterinária. Rio de Janeiro : Guanabara Koogan, 1996. Cap.15, p.142-143.

HELLYER, P.W.; GAYNOR, J.S. Acute postsurgical pain in dogs ans cats Compendium on Continuing Education for the Practing Veterinarian, v.20, n.2, p.145, 1998.

HEIDRICH, J.E.; KENT, G. Use of analgesia after surgery in animals. Journal of American Veterinary Medical Association, v.187, n.5, p.513-514, 1985.

LASCELlES, D.; WATERMAN, A. Analgesia in cats. In Practice, v.19, n.4, p.203-213, 1997

MAMA, K. New drugs in feline anesthesia. Compendium on Continuing Education for the Practing Veterinarian, v.20, n.2, p.131-132, 1998 .
McQUAY, H.I. Pre-emptive analgesia. British Journal of Anaesthesia, v.69, n.1, p.1-3, 1992.

PADDLEFORD, R.R. Manual of small animal anesthesia. 2 ed. Philadelphia : Saunders, 1999. Cap.2: Anesthetic agents. p.31-77.

SANFORD, J.; EWBANK, R.; MOLONY, V., et al. Guidelines for the recognition and assessment of pain in animals. Veterinary Record, v.118, n.12, p.334-338, 1986.

SHORT, C.E. Principles e practice of veterinary anesthesia. Baltimore : Williams and Wilkins, 1987. Cap.4: Pain, analgesics, and related medications: p.34-37.

SMITH, J.D.; ALLEN, S.W.; QUANDT, J.E., et al. Indicators of postoperative pain in cats and correlation with clinical criteria. American Journal of Veterinary Research, v.57, n.11, p.1674- 1678, 1996.

THURMON, J.C.; TRANQUILLI, W.J.; BENSON. G.J.. Lumb \& Jones'veterinary anesthesia. 3.ed. Baltimore : William \& Wilkins, 1996. Cap. 8: Preanesthetics and anesthetic adjuncts: p.183-209.

TRIM, C.M. Sedation and anesthesia. In: HOLZWORTH, J. Diseases of the cat, medicine e surgery. Philadelphia : Saunders, 1987. Cap.3. p.46-48.

WATTS, S.J.; SLOCOMBE, R.F.; HARBISON, W.D., $\boldsymbol{e} \boldsymbol{t} \boldsymbol{a l}$. Assessment of analgesia and other effects of morphine and thiambutene in mouse and cat. Australian Veterinary Journal, v.49, n.11, p.525-529, 1973.

WERNER, B.E.; TABOADA, J. Use of analgesics in feline medicine. Compendium on Continuing Education for the Practicing Veterinarian, v.16, n.4, p.493-499, 1994. 